\title{
Cannabidiol reduces lung injury induced by hypoxic-ischemic brain damage in newborn piglets
}

\author{
Luis Arruza ${ }^{1}$, Maria Ruth Pazos ${ }^{2}$, Nagat Mohammed ${ }^{2}$, Natalia Escribano ${ }^{3}$, Hector Lafuente ${ }^{4}$, Martín Santos ${ }^{2}$, \\ Francisco J. Alvarez-Díaz ${ }^{4}$, William Hind ${ }^{5}$ and Jose Martínez-Orgado ${ }^{1,2}$
}

BACKGROUND: Brain hypoxic-ischemic (HI) damage induces distant inflammatory lung damage in newborn pigs. We aimed to investigate the effects of cannabidiol (CBD) on lung damage in this scenario.

METHODS: Newborn piglets received intravenous vehicle, CBD, or CBD+WAY100635 (5- $\mathrm{HT}_{1 \mathrm{~A}}$ receptor antagonist) after $\mathrm{HI}$ brain damage (carotid flow interruption and $\mathrm{FiO}_{2} \quad 0.10$ for 30 min). Total lung compliance (TLC), oxygenation index (OI), and extravascular lung water content (EVLW) were monitored for $6 \mathrm{~h}$. Histological damage, interleukin (IL)-1 $\beta$ concentration, and oxidative stress were assessed in brain and lung tissue. Total protein content was determined in bronchoalveolar lavage fluid (BALF).

RESULTS: CBD prevented HI-induced deleterious effects on TLC and OI and reduced lung histological damage, modulating inflammation (decreased leukocyte infiltration and IL-1 concentration) and reducing protein content in BALF and EVLW. These effects were related to CBD-induced antiinflammatory changes in the brain. $\mathrm{HI}$ did not increase oxidative stress in the lungs. In the lungs, WAY100635 blunted the beneficial effects of CBD on histological damage, IL-1 concentration, and EVLW.

CONCLUSIONS: CBD reduced brain HI-induced distant lung damage, with $5-\mathrm{HT}_{1 \mathrm{~A}}$ receptor involvement in these effects. Whether the effects of CBD on the lungs were due to the antiinflammatory effects on the brain or due to the direct effects on the lungs remains to be elucidated.

$\mathbf{N}$ eonatal hypoxic-ischemic encephalopathy (NHIE) is often accompanied by damage of other organs in addition to the brain $(1,2)$. Current management of extracerebral impact of NHIE is limited to supportive therapy. The extra-cerebral impact of NHIE has been uniformly attributed to the redistribution of blood flow and/or the effects of global hypoxia-ischemia $(2,3)$. However, we have recently reported that cerebral hypoxic-ischemic (HI) and reperfusion injury in newborn piglets triggers a local inflammatory response that spreads to distant organs such as the lungs, where it induces inflammatory damage and respiratory dysfunction (4). Moreover, there is a positive correlation between interleukin (IL)-1 concentrations in the brain, plasma, and lung, suggesting a dose-effect relationship between cerebral HI and lung inflammation (4). The involvement of inflammation in lung damage after NHIE opens the possibility for new therapeutic approaches for this complication and may improve the outcome in these patients.

Cannabidiol (CBD) is a non-psychoactive cannabinoid with potent anti-inflammatory and anti-oxidant properties (5-8), which has demonstrated neuroprotective effects in newborn animal models of NHIE (8-13). CBD is a low-potency agonist of serotonin $5-\mathrm{HT}_{1 \mathrm{~A}}$ receptors (14). Activation of $5-\mathrm{HT}_{1 \mathrm{~A}}$ receptors is involved in multiple aspects of the neuroprotective qualities of CBD in piglet models of NHIE (12) and mouse models of adult stroke (15). Reduction of HI-induced brain damage in newborn animals is related to modulation of inflammation and oxidative stress $(8,10,12,13)$. The antiinflammatory properties of CBD have also been demonstrated in extra-cerebral organs. In this regard, $\mathrm{CBD}$ has been shown to reduce inflammation in animal models of arthritis, intestinal hypermotility, and diabetes. Interestingly, CBD has recently been shown to decrease lung inflammation in a murine model of lipopolysaccharide (LPS)-induced lung damage $(16,17)$.

The aim of this study was to investigate and characterize the effects of CBD on NHIE-related inflammatory lung damage in newborn piglets.

\section{RESULTS}

\section{General Characteristics and Hemodynamic Status}

After randomization, the number of animals per group was $n=6$ (sham operation (SHM)), HV $n=8$ (vehicle (HV)), $n=9\left(\mathrm{CBD}\right.$ alone (HC)), and $n=6$ (together with $5-\mathrm{HT}_{1 \mathrm{~A}}$ receptor antagonist WAY100635 (HCW)). Three piglets died during $\mathrm{HI}$; one piglet from the $\mathrm{HC}$ group died $2 \mathrm{~h}$ after $\mathrm{HI}$ because of massive myocardial ischemia. This is a frequent complication in this model and appeared too early after CBD administration to be considered a drug side effect. At the time of the experiment, piglet age was $1.80 \pm 0.17,1.7 \pm 0.2$,

\footnotetext{
${ }^{1}$ Department of Neonatology, Hospital Clínico San Carlos-IdISSC, Madrid, Spain; ${ }^{2}$ Health Research Institute Puerta de Hierro Majadahonda, Madrid, Spain; ${ }^{3}$ Department of Pathology, Hospital Clínico San Carlos, Madrid, Spain; ${ }^{4}$ Intensive Care Translational Research Group, Biocruces Health Research Institute, Bizkaia, Spain; ${ }^{5}$ GW Research, Cambridge, UK. Correspondence: Jose Martinez-Orgado (jose.martinezo@salud.madrid.org)

Received 2 February 2017; accepted 30 March 2017; advance online publication 3 May 2017. doi:10.1038/pr.2017.104
} 
Table 1. Homeostatic parameters

\begin{tabular}{|c|c|c|c|c|}
\hline Parameter & $\operatorname{SHM}(n=6)$ & $\mathrm{HV}(n=8)$ & $\mathrm{HC}(n=10)$ & $\mathrm{HCW}(n=6)$ \\
\hline \multicolumn{5}{|c|}{ MABP $(\mathrm{mmHg})$} \\
\hline B & $76.3(7)$ & $80.8(3)$ & $80.5(3)$ & $74.1(3.1)$ \\
\hline 1 & $83.5(6)$ & $56.4(8)^{*}$ & $60.3(3)^{*}$ & $59.0(8.6)^{*}$ \\
\hline $\mathrm{D}$ & $88.5(5.8)$ & $86.0(8.6)$ & $90.2(3.5)$ & $83.0(5.3)$ \\
\hline E & $78.8(5)$ & $59.6(3)^{*}$ & $78.8(3)^{\#}$ & $54.4(7.5)^{*}, \S$ \\
\hline \multicolumn{5}{|c|}{$\mathrm{CO}(\mathrm{ml} / \mathrm{min}$ per $100 \mathrm{~g})$} \\
\hline B & $318.4(29)$ & $356.9(45)$ & $357.1(24)$ & $311.7(28)$ \\
\hline I & $340.4(24)$ & 355.7 (34) & $321.1(43)$ & $360.3(31)$ \\
\hline $\mathrm{D}$ & $335.2(27)$ & $398.9(61)$ & $382.1(52)$ & $363.9(22)$ \\
\hline$E$ & $350.2(36)$ & $344.3(42)$ & $335.3(30)$ & $295.9(42)$ \\
\hline \multicolumn{5}{|l|}{$p H$} \\
\hline B & $7.34(0.02)$ & $7.32(0.02)$ & $7.36(0.02)$ & $7.35(0.01)$ \\
\hline 1 & $7.38(0.02)$ & $7.17(0.03)^{*}$ & $7.19(0.03)^{*}$ & $7.20(0.01)^{*}$ \\
\hline $\mathrm{D}$ & $7.38(0.01)$ & $7.21(0.02)^{*}$ & $7.20(0.05)^{*}$ & $7.23(0.01)^{*}$ \\
\hline$E$ & $7.38(.01)$ & $7.32(0.04)$ & $7.32(0.02)$ & $7.30(0.03)$ \\
\hline \multicolumn{5}{|l|}{$\mathrm{P}_{a} \mathrm{O}_{2}$} \\
\hline B & $74.2(6.5)$ & $82.1(6.2)$ & $80.2(3.9)$ & $74.8(4.6)$ \\
\hline I & $73.8(2.0)$ & $32.0(6.1)^{*}$ & $25.3(2.6)^{*}$ & $27.4(2.6)^{*}$ \\
\hline $\mathrm{D}$ & $73.0(2.3)$ & $73.8(4.2)$ & $78.5(3.9)$ & $77.4(2.7)$ \\
\hline E & $72.2(5.5)$ & $71.1(5.1)$ & $75.6(3.2)$ & $73.8(2.0)$ \\
\hline \multicolumn{5}{|c|}{$p \mathrm{CO}_{2}(\mathrm{mmHg})$} \\
\hline B & $41.3(2.4)$ & $39.4(2.6)$ & $40.7(1.4)$ & $39.9(1.8)$ \\
\hline I & $39.4(2.4)$ & $39.1(2.6)$ & $40.9(2.3)$ & $38.4(0.9)$ \\
\hline $\mathrm{D}$ & $39.3(2.3)$ & $42.4(2.2)$ & $44.4(2.3)$ & $41.1(1.6)$ \\
\hline E & $38.8(1.1)$ & $42.2(2.9)$ & $42.3(1.6)$ & $39.6(2.5)$ \\
\hline \multicolumn{5}{|l|}{$\mathrm{SpO}_{2}(\%)$} \\
\hline B & $96.6(0.8)$ & $96.2(1.1)$ & $96.7(0.9)$ & $96.5(1.0)$ \\
\hline 1 & $96.1(0.8)$ & $26.2(4.0)^{*}$ & $26.7(7.2)^{*}$ & $24.8(3.9)^{*}$ \\
\hline D & $96.1(2.2)$ & $83.2(9.5)$ & $82.6(8.1)$ & $90.1(8.6)$ \\
\hline$E$ & $98.2(1.1)$ & $96.7(0.7)$ & $95.3(0.6)$ & $95.4(0.6)$ \\
\hline \multicolumn{5}{|c|}{$\mathrm{rsSO}_{2}(\mathrm{~S})(\%)$} \\
\hline B & $55.0(2.7)$ & $56.2(5.2)$ & $52.2(2.5)$ & $53.6(3.1)$ \\
\hline 1 & $55.0(2.7)$ & $24.1(2.7)^{*}$ & $26.2(4.1)^{*}$ & $22.6(3.3)^{*}$ \\
\hline D & $52.2(2.6)$ & $56.0(4.9)$ & $54.3(4.4)$ & $53.6(2.2)$ \\
\hline E & $51.1(2.5)$ & $52.6(5.6)$ & $57.0(6.0)$ & $57.6(3.4)$ \\
\hline
\end{tabular}

B, baseline; CO, cardiac output; D, drug administration; $E$, end of observation period; I, end of $\mathrm{HI}$ insult; $\mathrm{MABP}$, mean arterial blood pressure; $\mathrm{rsSO}_{2}$, somatic regional oxygen saturation; $\mathrm{SpO}_{2}$, oxygen saturation.

Data are expressed as mean (s.e.). ${ }^{*} P<0.05$ vs. $S H M$; ${ }^{\#} P<0.05$ vs. $H V$; ${ }^{5} P<0.05$ vs. $\mathrm{HC}$. All were assessed by the Kruskall-Wallis test for range analysis with Dunn's post hoc test for multiple comparisons.

$1.9 \pm 0.1$, and $1.8 \pm 0.2$ days for SHM, HV, HC, and HCW, respectively (no significance). Weight at that time was $1.9 \pm 0.1,1.7 \pm 0.1,1.8 \pm 0.1$, and $2.0 \pm 0.1 \mathrm{~kg}$ for SHM, HV, $\mathrm{HC}$, and HCW, respectively (no significance).
Table 2. Hypoxic-ischemic brain damage

\begin{tabular}{ccccc}
\hline Parameter & $\mathrm{SHM}(n=6)$ & $\mathrm{HV}(n=8)$ & $\mathrm{HC}(n=10)$ & $\mathrm{HCW}(n=6)$ \\
\hline \multicolumn{2}{l}{ aEEG basal amplitude $(\mu \mathrm{V})$} & & & \\
B & $16.0(2.1)$ & $15.8(2.0)$ & $14.8(1.9)$ & $11.2(1.5)$ \\
I & $16.6(2.5)$ & $2.5(0.5)^{*}$ & $1.9(0.2)^{*}$ & $1.4(0.2)^{*}$ \\
D & $16.6(2.4)$ & $4.1(1.0)^{*}$ & $4.1(0.8)^{*}$ & $3.0(0.8)^{*}$ \\
E & $11.8(1.6)$ & $3.4(0.8)^{*}$ & $7.7(1.5)^{*, \#}$ & $2.8(0.2)^{*, \text {, }}$
\end{tabular}

aEEG mean amplitude $(\mu \mathrm{V})$

$\begin{array}{lllll}\text { B } & 21.0(2.0) & 21.0(2.4) & 20.1(2.9) & 19.4(1.9) \\ \text { I } & 21.6(2.2) & 2.8(0.6)^{*} & 2.4(0.2)^{*} & 2.0(0.2)^{*} \\ \text { D } & 21.1(1.7) & 5.4(1.1)^{*} & 5.7(1.2)^{*} & 4.5(1.1)^{*} \\ \text { E } & 17.3(1.4) & 3.8(0.8)^{*} & 12.1(2.8)^{*, \#} & 2.9(0.8)^{*, \$}\end{array}$

Dead neurons (Nissl) (\%)

$\begin{array}{ccccc}\mathrm{E} & 5.1(1.7) & 20.1(3.8)^{*} & 9.1(2.9)^{\#} & 17.9(2.9)^{*}, \mathrm{~S} \\ \mathrm{rCSO}_{2}(\%) & & & & \\ \mathrm{B} & 55.0(2.7) & 52.6(1.8) & 49.6(3.7) & 51.6(3.1) \\ \mathrm{I} & 55.1(2.7) & 19.1(3.3)^{*} & 16.8(0.9)^{*} & 16.5(3.3)^{*} \\ \mathrm{D} & 49.8(2.4) & 51.0(2.2) & 45.6(6.3) & 48.5(2.9) \\ \mathrm{E} & 54.0(2.7) & 50.6(1.3) & 48.0(2.5) & 47.3(2.6)\end{array}$

IL-1 $(p g / m l)$

E $\quad 111.7(9.5) \quad 137.6(10.1)^{*} \quad 120.1(4.4)^{\#} \quad 130.2(2.2)^{*}$, s

Protein carbonylation (NDS)

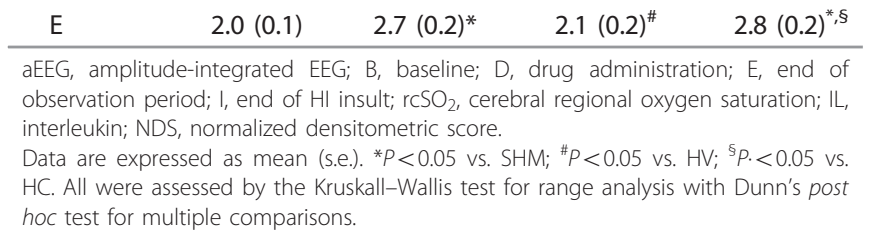

The hemodynamic status of piglets from the different study groups is shown in Table 1. HI led to a transient reduction in mean arterial blood pressure (MABP), which recovered by the time treatment was administered in all groups. By the end of the experiment, the MABP decreased further in HV animals to a level that was significantly lower than SHM, despite the fact that $50 \%$ of $\mathrm{HV}$ piglets received dopamine infusion to maintain normal MABP $(n=4$, mean dose $13.1 \pm 4.1 \mu \mathrm{g} / \mathrm{kg} /$ min). CBD appeared to have a beneficial effect on MABP, as none of the $\mathrm{HC}$ animals required dopamine infusion and MABP returned to SHM-like levels after CBD administration. However, coadministration of CBD with WAY100635 reversed the beneficial effect of $\mathrm{CBD}$, where the MABP in HCW animals was lower than that of SHM or HC animals by the end of the experiment, despite $66 \% \mathrm{HCW}$ piglets receiving dopamine $(n=4$, mean dose $11.2 \pm 1.2 \mu \mathrm{g} / \mathrm{kg} / \mathrm{min})$. Changes in MABP had no effect on cardiac output, which remained similar to SHM throughout the experiment in all groups. In accordance with this, regional hemoglobin oxygen saturation $\left(\mathrm{rSO}_{2}\right)$ always remained similar to SHM except during $\mathrm{HI}$ insult. 


\section{$C B D$ and brain-derived lung injury $\mid$ Articles}

\section{Brain Damage}

$\mathrm{HI}$ led to an immediate fall in brain electrical activity that was maintained until the end of the experiment (Table 2). Postinsult administration of $\mathrm{CBD}$ resulted in a progressive improvement in amplitude-integrated electroencephalography amplitude, so that by the end of the experiment it was significantly higher than in HV animals (Table 2), although it did not return to baseline values. Administration of WAY100635 completely inhibited the effect of CBD on cerebral electrical activity, with basal and mean amplitudeintegrated electroencephalography amplitudes being similar to $\mathrm{HV}$ animals.

Nissl-stained brain tissue analysis revealed a reduced number of normal neurons in HI animals as compared with SHM animals, with a fourfold increase in the percentage of nonviable neurons in the frontoparietal cortex (Table 2). CBD treatment prevented such an increase in the death of neurons. Coadministration of WAY100635 inhibited the protective effect of CBD (Table 2).

Cerebral near-infrared spectroscopy did not reveal differences among groups except during HI insult, where cerebral regional oxygen saturation $\left(\mathrm{rCSO}_{2}\right)$ declined in all groups (Table 2).

Cerebral HI induced a local inflammatory response as evidenced by an increase in IL- 1 concentration in brain cortex tissue in HC animals compared with SHM animals (Table 2). CBD treatment prevented HI-induced increase in IL-1 brain concentration (Table 2), an effect that was attenuated by the coadministration of WAY100635 (Table 2).

$\mathrm{HI}$ also increased oxidative stress as shown by the increase in protein carbonylation in $\mathrm{HV}$ animals as compared with SHM animals (Table 2). CBD treatment prevented HIinduced increase in brain oxidative stress (Table 2), an effect that was attenuated by the coadministration of WAY100635 (Table 2).

\section{Lung Damage}

Lung mechanics and gas exchange. HI insult led to a progressive impairment in gas exchange as evidenced by the increase in oxygenation index (OI) in HV animals as compared with SHM animals (Figure 1a). Gas-exchange impairment, however, was limited to oxygen, as $\mathrm{pCO}_{2}$ remained similar in SHM and HV animals throughout the experiment (Table 1). In addition, HI led to a gradual decrease in total lung compliance (TLC; Figure 1b). Such effects of HI on the lungs were not associated with a particular increase in lung edema, as extravascular lung water content (EVLW) was similar in $\mathrm{HV}$ and SHM animals throughout the experiment (Figure 1c).

Administration of CBD after the $\mathrm{HI}$ insult led to remarkably beneficial effects in the lungs, as changes in either OI or $\mathrm{pCO}_{2}$ were not observed in $\mathrm{HC}$ animals (Figure 1a; Table 2). In addition, the HI-induced drop in TLC was reversed in $\mathrm{HC}$ animals from $3 \mathrm{~h}$ after $\mathrm{CBD}$ administration (Figure 2b). Furthermore, CBD treatment after HI led to a gradual decrease in lung edema, as shown by the decrease in EVLW to levels that were similar to baseline, which became
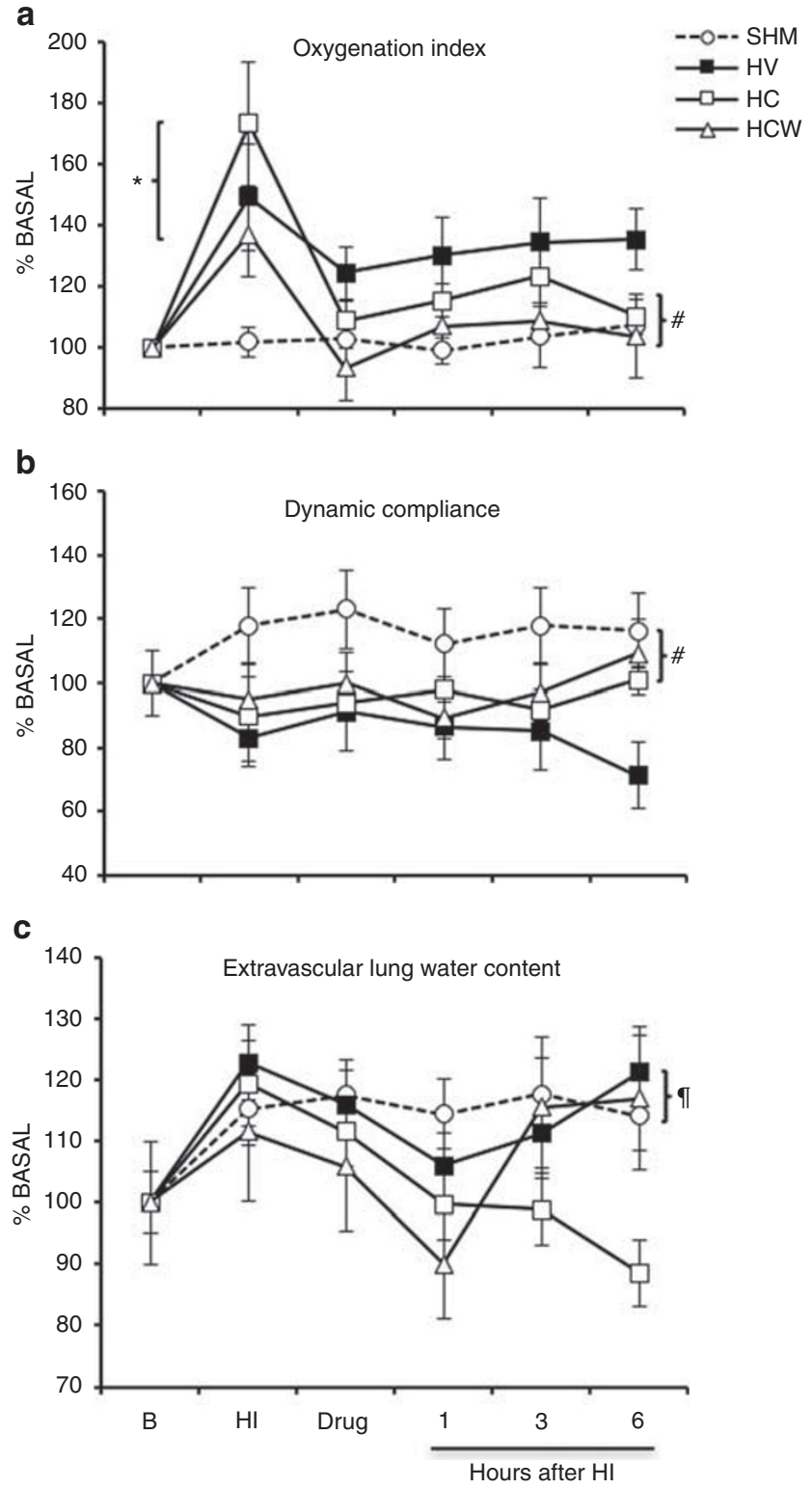

Figure 1. Lung mechanics and blood exchange. Changes in (a) oxygenation index (OI), (b) total lung compliance (TLC), and (c) extravascular lung water content (EVLW) in 1-2-day-old piglets after sham operation (SHM) or after cerebral hypoxic-ischemic insult and treatment with vehicle (HV), $\mathrm{CBD}$ alone $(\mathrm{HC})$, or together with WAY100635 (HCW). Results are expressed as mean \pm s.e.m. of 6-10 animals. B, basal; $\mathrm{HI}$, hypoxic-ischemic insult. ${ }^{*} P<0.05$ vs. SHM; ${ }^{\#} P<0.05$ vs. HV; ${ }^{n} P<0.05$ vs. HC. All were assessed by the Kruskall-Wallis test for range analysis with Dunn's post hoc test for multiple comparisons.

significantly different even from SHM by the end of the experiment (Figure 1c).

Coadministration of WAY100635 did not modify the CBD effects on gas exchange or TLC (Figure 1a,b; Table 2), but inhibited the effect of CBD on EVLW (Figure 1c).

Histological lung damage. Changes in lung mechanics and gas exchange were associated with histologically apparent lung damage, as evidenced by the higher pathology score in $\mathrm{HV}$ 


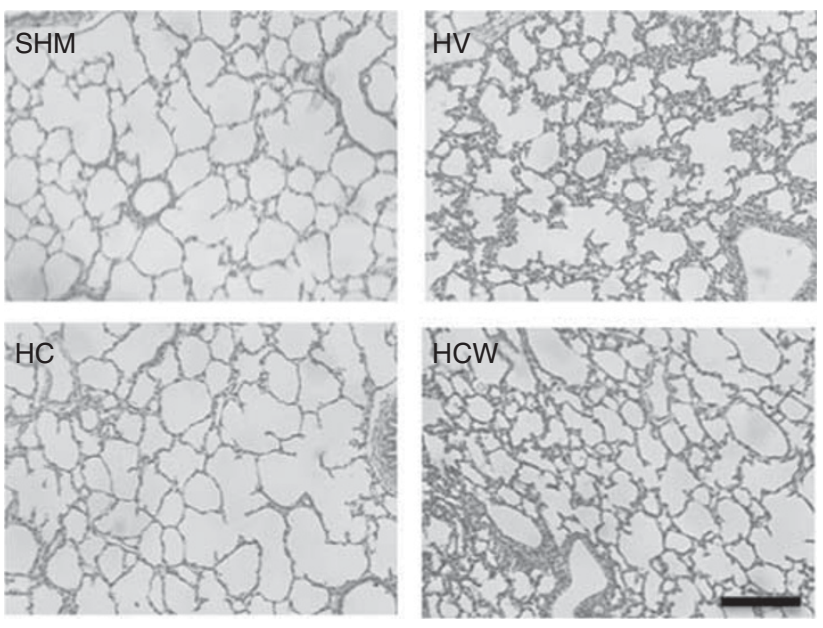

Lung histological damage

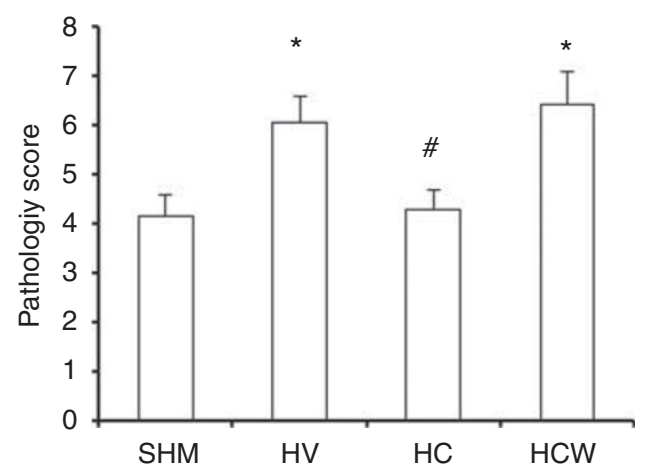

Figure 2. Histologic lung damage. Top: representative light microphotographs of hematoxylin-eosin staining of lung tissue sections, obtained from 1-2-day-old piglets after sham operation (SHM) or after cerebral hypoxic-ischemic insult and treatment with vehicle (HV), CBD alone $(\mathrm{HC})$, or together with WAY100635 (HCW). In the lung from HV, interstitial leukocyte infiltration, congestive hyperemia, and interstitial edema were observed, which were reduced by CBD treatment. WAY100635 reversed the protective effects of CBD. Original magnification $\times 200$, bar: $200 \mu \mathrm{m}$. Bottom: quantification of lung damage by a severity score. Results are expressed as mean \pm s.e.m. of 6-10 animals. B, basal; $\mathrm{HI}$, hypoxic-ischemic insult. ${ }^{*} P<0.05$ vs. SHM; ${ }^{\#} P<0.05$ vs. HV. All were assessed by the Kruskall-Wallis test for range analysis with Dunn's post hoc test for multiple comparisons.

animals compared with SHM animals (Figure 2). The lesion consisted mostly of interstitial leukocyte infiltration, congestive hyperemia, and interstitial edema. HI-induced histological damage was not observed in $\mathrm{HI}$ animals treated with $\mathrm{CBD}$ (Figure 2). The beneficial effect of CBD was prevented by concomitant administration of WAY100635 (Figure 2).

Lung inflammation. HI led to an increase in total protein content in bronchoalveolar lavage fluid (BALF), as observed in HV animals compared with SHM animals (Figure 3a). Such an increase was associated with an increase in lung IL-1 concentration in HV (Figure 3b). Treatment with CBD inhibited those responses so that no differences were found in either protein content in BALF or lung IL-1 concentration between HC and SHM animals (Figure 3). Coadministration of

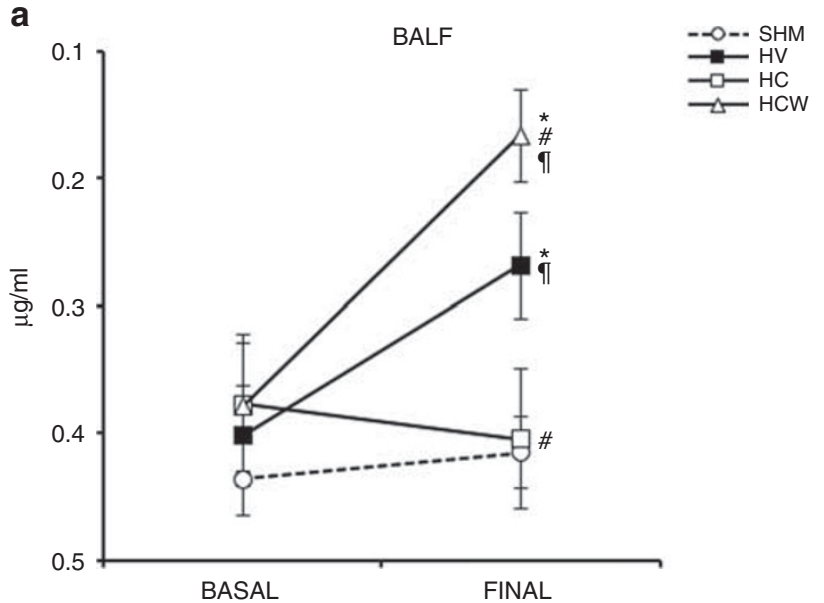

b

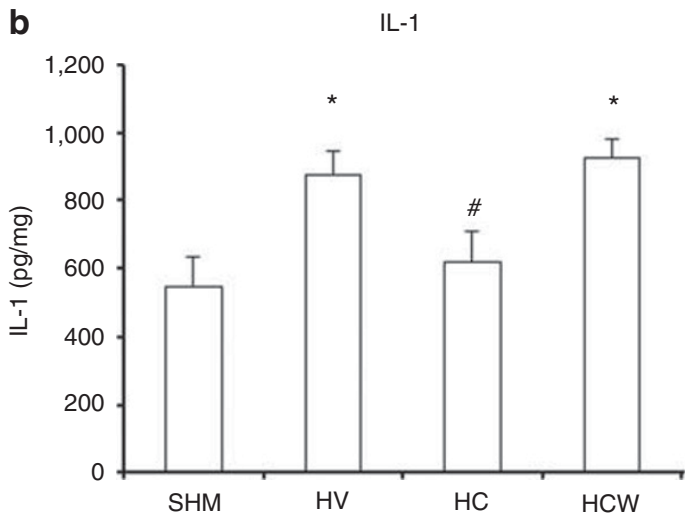

Figure 3. Assessment of lung inflammation. (a) Changes in bronchoalveolar lavage fluid (BALF) protein content before $\mathrm{HI}$ insult (basal) and at the end of the experiment (final) and (b) determination of interleukin-1 (IL-1) concentration in lung tissue in 1-2-day-old piglets after sham operation (SHM) or after cerebral hypoxic-ischemic insult and treatment with vehicle (HV), CBD alone $(\mathrm{HC})$, or together with WAY100635 (HCW). Results are expressed as mean \pm s.e.m. of 6-10 animals. ${ }^{*} P<0.05$ vs. SHM; ${ }^{*} P<0.05$ vs. HV; ${ }^{~} P<0.05$ vs. HC. All were assessed by the Kruskall-Wallis test for range analysis with Dunn's post hoc test for multiple comparisons.

WAY100635 completely eliminated the beneficial effect of $\mathrm{CBD}$ on lung IL-1 concentration (Figure $3 \mathbf{b}$ ). Strikingly, coadministration of WAY100635 not only reversed the beneficial effect of CBD on protein content in BALF but also led to an even greater increase in such content than that observed in HV animals (Figure 3a).

Protein carbonylation in the lung. No significant differences were found among the different groups for this marker of oxidative stress (Figure 4).

\section{DISCUSSION}

The results presented in this work have a twofold value. First, they support our previous study, demonstrating that cerebral $\mathrm{HI}$ in piglets triggers an inflammatory response not merely confined to the brain, but spreads to distant organs such as the lung where it induces inflammatory damage and 


\section{$C B D$ and brain-derived lung injury $\mid$ Articles}

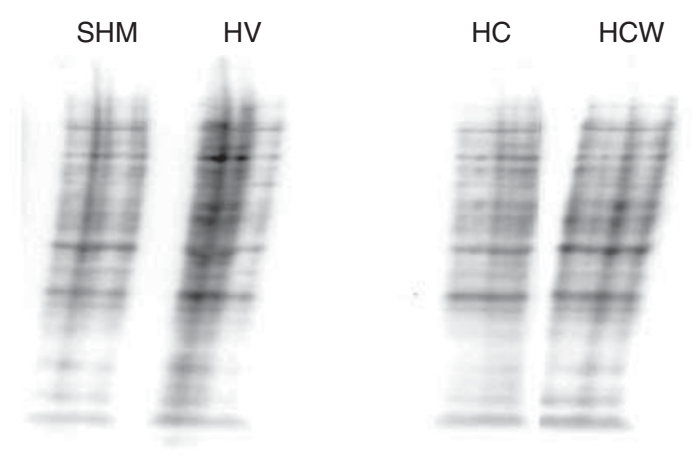

Protein carbonylation

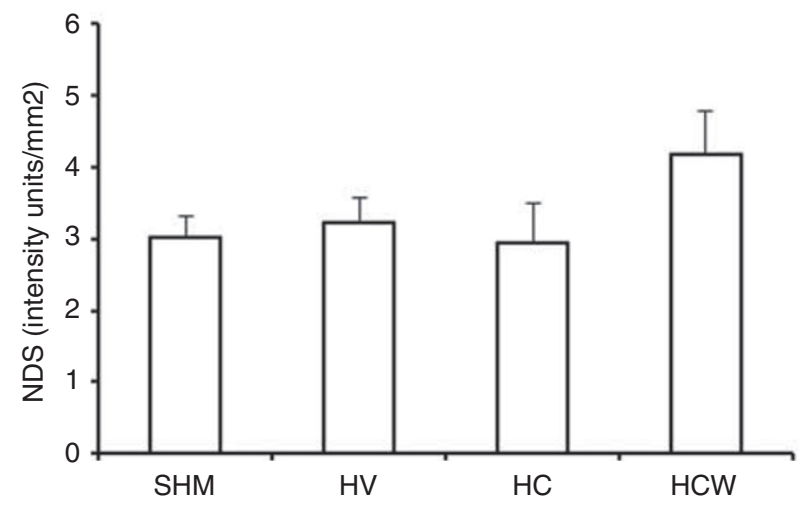

Figure 4. Assessment of oxidative stress in lungs. Top: representative western blot probed with antibody to derived protein carbonyl side groups (OxyBlot), performed on brain samples from 1-2-day-old piglets after sham operation (SHM) or after cerebral hypoxic-ischemic insult and treatment with vehicle (HV), CBD alone (HC), or together with WAY100635 (HCW). Bottom: densitometric analysis of relative protein carbonyl contents. Results are expressed as mean \pm s.e.m. of 6-10 animals.

respiratory dysfunction (4). Second, we demonstrate that administration of CBD post insult has beneficial effects on remote inflammatory lung injury appearing after a cerebral $\mathrm{HI}$ insult, an effect not previously reported.

The inflammatory lung damage appearing after $\mathrm{HI}$ in newborn pigs is illustrated by the presence of increased infiltration by leukocytes and elevated cytokine concentration in lung tissue, in addition to increased vascular permeability as shown by the increase in total protein content in BALF (4). All these findings were reproduced in this work. In the current study, post-insult administration of CBD improved lung mechanics and gas exchange, and reduced histological damage. To the best of our knowledge, the only precedent in the literature that demonstrated a benefit of CBD on distant lung damage in newborn animals was from our research group (9). The study was performed in a similar model of $\mathrm{HI}$ in piglets where the observed respiratory dysfunction was attributed to a ventilator-induced lung injury (9). In this case, administration of CBD at a dose $(0.1 \mathrm{mg} / \mathrm{kg})$ lower than that in our current work produced an unexpected improvement in TLC and gas exchange, attributed to an anti-inflammatory action of CBD on the lung (9). The current study confirmed that the beneficial effects of $\mathrm{CBD}$ on the lung after HI were related to decreased leukocyte infiltration and IL-1 concentration in lung tissue, and protein content in BALF. Our results represent new evidence supporting the well-known strong anti-inflammatory nature of CBD (6-8).

The question, which was not answered directly in this work, is whether the reduction in inflammatory lung damage by CBD is the result of a decrease in brain inflammation or a direct anti-inflammatory effect on the lungs. In this work, we observed that $\mathrm{CBD}$ administration reduced $\mathrm{HI}$-induced brain damage, and that this neuroprotective effect was related to the modulation of brain cytokine production, as previously described $(8,10-13)$. We have reported, in a model identical to this one, that cytokine concentration in the lungs is directly related to cytokine concentration in the brain, which accounts for the distant cerebral origin of the lung damage (4). Thus, the protective anti-inflammatory effect of $\mathrm{CBD}$ on the brain after $\mathrm{HI}$ insult could likely result in a reduced inflammatory repercussion in the lungs. In an adult mouse model of stroke, damaged brain neurons release the HMGB1 (high mobility group box 1) protein, which activates monocytes increasing cytokine production and inducing inflammatory damage in the brain (18) and extra-cerebral organs such as heart (19) and lung (20) in proportion to the extent of brain damage. Thus, neuroprotectants such as CBD might reduce systemic inflammatory response by reducing the extent of post-HI brain damage. Indeed, following adult stroke in mice CBD has been shown to decrease the number of microglial cells expressing HMGB1-positive cell and HMGB1 levels in plasma, and this was associated with a significant increase in survival (21). We cannot rule out, however, that CBD was also exerting a direct effect on the lungs. Ultimately, whether the CBD-induced anti-inflammatory effects on the lungs were direct or not is of little consequence in this disease model, as the decrease in lung inflammation is beneficial irrespective of its site of action. CBD has also demonstrated the beneficial anti-inflammatory effects in animal models of LPS-induced lung injury and of asthma $(16,17,22)$. Moreover, and in accordance with our results, vascular permeability is also reduced in LPS-induced lung injury in mice, as evidenced by a lower protein content in BALF in treated animals (16). Interestingly, reducing lung inflammation has been demonstrated to exert neuroprotective effects in rats receiving intratracheal mesenchymal stem cells after hyperoxia-induced lung damage (23). Whether the extra-cerebral anti-inflammatory effects of CBD could account for the neuroprotection of CBD in our model deserves further research.

CBD treatment prevented HI-induced increase of EVLW. In this model, such an increase has been attributed to inflammatory lung edema (4). Therefore, the effect of CBD could account for its anti-inflammatory effects. However, brain damage-induced increase of EVLW is also associated with sympathetic hyperactivity due to brain damage (20). Thus, the effect of CBD on EVLW could account for its neuroprotective effect as well. Increased EVLW affects lung elastance (20). The effects of CBD on TLC could then rely 


\section{Articles | Arruza et al.}

at least partly on its effects on EVLW. Although it could improve lung mechanics, drying lungs may have deleterious effects by reducing cardiac output (24). This was not the case in our model as CBD-treated animals show normal cardiac output.

Another mechanism involved in the beneficial effects of CBD after $\mathrm{HI}$ is the modulation of oxidative stress $(5-7,12,13)$. We could not demonstrate the involvement of such a mechanism in the beneficial effect of $\mathrm{CBD}$ on the lungs in this work because we were unable to detect an increase of oxidative stress in the lungs after HI. We were very careful to avoid hyperoxygenation in ventilated piglets, as shown by oxygen saturation $\left(\mathrm{SpO}_{2}\right)$ and $\mathrm{rSO}_{2}$ monitoring data, and this may contribute to the lack of oxidative stress in the lungs. In the absence of hyperoxygenation, a major source of oxidative stress after $\mathrm{HI}$ is inflammation (25). Thus, we detected increased oxidative stress in parallel to increased cytokine concentration in the brain, the primary location where the HI insult took place. The finding of a lack of similar increase in oxidative stress in the lungs may account for a distal-cerebral origin of lung inflammation. We cannot rule out, however, that oxidative stress could subsequently increase in lungs to detectable levels after $6 \mathrm{~h}$. Therefore, longer follow-up studies would be interesting.

A striking result was that $5-\mathrm{HT}_{1 \mathrm{~A}}$ antagonism reversed the beneficial effects of CBD in functional, histological, and biochemical studies. CBD is a low-potency agonist of 5- $\mathrm{HT}_{1 \mathrm{~A}}$ receptors (14). This mechanism is involved in the majority of the neuroprotective effects (including anti-inflammatory, anti-oxidant, and anti-excitotoxic) of CBD, as demonstrated in newborn pigs after an HI insult (12) and adult mice after stroke (15). It is unclear, however, whether those effects are the result of a direct action of CBD or the improvement of brain circulation after the ischemic insult, as demonstrated in adult mice after stroke (15). Although central $5-\mathrm{HT}_{1 \mathrm{~A}}$ receptors are involved in cardiorespiratory control, in particular, central chemoreception, and respiratory rythmogenesis $(26,27)$, there are no reports regarding the involvement of peripheral $5-\mathrm{HT}_{1 \mathrm{~A}}$ receptors on lung pathophysiology. Therefore, the blunting of lung beneficial effects of CBD by WAY100635 coadministration might be due to reversion of the neuroprotective effects of CBD. This finding would then support the protective effect of CBD in the lung after $\mathrm{HI}$ being more related to its central anti-inflammatory or neuroprotective effect, rather than a direct effect on the lungs. Interestingly, the 5- $\mathrm{HT}_{1 \mathrm{~A}}$ receptor agonist buspirone enhances vagal responses in anesthetized rabbits, with WAY100635 inhibiting such effects but not eliciting effects by itself (28). Whether CBD could enhance vagal responses via $5-\mathrm{HT}_{1 \mathrm{~A}}$ receptor activation is currently unknown and deserves further research.

Administration of CBD prevented the $\mathrm{HI}$-induced decrease on MABP. However, we observed that adding WAY100635 to $\mathrm{CBD}$ led to a decrease in MABP, so that the majority (four out of six) of HCW piglets needed dopamine infusion to avoid severe hypotension. WAY100635 is known to reduce blood pressure at least in part because it also blocks $\alpha 1$ adrenoreceptors (29). It is unlikely that this cardiovascular effect had a relevant influence in WAY100635-induced blunting of CBD protective effects in the lungs as the drop in MABP had no hemodynamic deleterious effect, as shown by the lack of influence on cardiac output or regional blood flow. The inhibitory effects of WAY100635 coadministration on the protective effects of CBD on the lungs were observed in the histological studies, EVLW evolution, and lung cytokine concentration, but not in TLC or OI evolution. This suggests that the effects of CBD on lung mechanics and gas exchange were not only due to modulation of the inflammatory condition after $\mathrm{HI}$ but also due to other yet-to-be determined mechanisms. It has been reported that CBD has bronchodilator effects in guinea pigs exposed to exogenous spasmogens (30), but there is no information supporting such an effect in other conditions.

In conclusion, post-insult administration of CBD reduced brain HI-induced distant lung damage, as demonstrated by functional and histology studies. Whether the beneficial effect of CBD was due to its favorable anti-inflammatory effect on the brain, a direct effect on lung inflammation, or other mechanisms not yet reported remains to be elucidated. Activation of serotonin $5-\mathrm{HT}_{1 \mathrm{~A}}$ receptors was involved in the beneficial effects of CBD on the lungs. These results pave the way for new perspectives regarding alternative therapeutic approaches for lung complications in infants suffering from NHIE, which could result in an improved outcome.

\section{METHODS}

The experimental protocol met European and Spanish regulations for the protection of experimental animals (86/609/EEC and RD 53/2013) and was approved by the Ethics Committee for Animal Welfare from University Hospital Puerta de Hierro Majadahonda (Madrid, Spain). Sample size calculation was based on the minimum number of animals necessary to attain statistical significance.

\section{Animal Preparation}

The piglet model of HI was used for the experiments as previously reported $(4,12,13)$. In short, $1-2$-day-old male Long White-Landrace piglets were intubated and mechanically ventilated (Evita 4, Dräger, Germany) under sedo-analgesia and paralysis (propofol $12 \mathrm{mg} / \mathrm{kg} / \mathrm{h}$, rocuronium $0.6 \mathrm{mg} / \mathrm{kg} / \mathrm{h}$, and morphine chloride $0.1 \mathrm{mg} / \mathrm{kg} / 3 \mathrm{~h}$ ). Initial ventilator settings (positive end-expiratory pressure $5 \mathrm{~cm} \mathrm{H}_{2} \mathrm{O}$, respiratory rate 30 b.p.m., inspiratory time $0.5 \mathrm{~s}$, and $\mathrm{FiO}_{2} 0.21$ ) were adjusted during the experiment to maintain a tidal volume of $6 \mathrm{ml} / \mathrm{kg}, \mathrm{SpO}_{2}$ 90-95\%, and $\mathrm{PaCO}_{2} 35-45 \mathrm{~mm} \mathrm{Hg}$. Tidal volume, mean airway pressure, and TLC were continuously measured with the ventilator's computerized pneumotachographer. End-tidal carbon dioxide concentration and $\mathrm{SpO}_{2}$ were continuously measured (Ohmeda 5250 RGM, Louisville, CO). Both carotid arteries were exposed and surrounded by an elastic band, and a right jugular vein indwelling catheter was inserted to infuse $4 \mathrm{mg} / \mathrm{kg} / \mathrm{min}$ dextrose and sedation. Cardiac output, MABP, EVLW content, and central temperature were monitored (PiCCO Plus, Pulsion, Feldkirchen, Germany) by a femoral artery indwelling catheter (Ominare CMS24, Philips Healthcare, Eindhoven, Netherlands) at baseline, at the end of hypoxia-ischemia (HI), and hourly throughout the experiment. Any drop in MABP below $30 \mathrm{~mm} \mathrm{Hg}$ prompted the infusion of dopamine $(5-20 \mu \mathrm{g} / \mathrm{kg} / \mathrm{min})$. Body temperature was maintained at $37.5-38^{\circ} \mathrm{C}$ by an air-warmed blanket (Bair Hugger, Agustine Medical, Eden Prairie, MN). OI (mean airway pressure $\times \mathrm{FiO}_{2} /$ 


\section{CBD and brain-derived lung injury $\mid$ Articles}

$\mathrm{PaO}_{2} \times 100$ ) was calculated at baseline, after $\mathrm{HI}$, and hourly throughout the experiment. $\mathrm{rSO}_{2}$ was continuously measured by near-infrared spectroscopy (Invos 5100C, Medtronics, Minneapolis, $\mathrm{MN}$, USA) with neonatal cerebral $\left(\mathrm{rCSO}_{2}\right)$ and somatic (somatic regional oxygen saturation, $\mathrm{rsSO}_{2}$ ) sensors (OxyAlert NIRSensor, Medtronics, Minneapolis, MN, USA). Finally, brain electrical activity was monitored by amplitude-integrated electroencephalography (BRM3, BrainZ Instruments, Auckland, NZ).

\section{Experimental Protocol}

HI was induced by pulling out the elastic bands until complete interruption of carotid blood flow and by lowering $\mathrm{FiO}_{2}$ to 0.10 . HI lasted for $30 \mathrm{~min}$ starting from when $\mathrm{rcSO}_{2}$ fell below $20 \%$ and amplitude-integrated electroencephalography mean amplitude declined under $5 \mu \mathrm{V}$. Afterward, carotid blood flow was restored and $\mathrm{FiO}_{2}$ returned to 0.21 . Thirty minutes after the end of $\mathrm{HI}$, animals were randomly assigned to receive a single intravenous dose of $\mathrm{HV}$ (ethanol:solutol:saline 2:1:17), $1 \mathrm{mg} / \mathrm{kg}$ of $\mathrm{HC}$, or $\mathrm{HCW}$ $0.1 \mathrm{mg} / \mathrm{kg}$. A set of HI piglets $(n=4)$ received WAY100635 alone and were assessed for its potential effects on OI, TLC, EVLW, and BALF. These piglets were not included in the analysis because there were no differences between these and HV piglets. CBD (GW Research, Cambridge, UK) was prepared from a $5 \mathrm{mg} / \mathrm{ml}$ solution of CBD in ethanol:solutol:saline $(2: 1: 17)$ and further dissolved in saline. Non-HI piglets that were similarly managed served as controls (SHM). Six hours after HI or the equivalent period in SHM, piglets were killed by intravenous potassium chloride bolus. Brain and lungs were then collected, as reported elsewhere $(4,12)$.

\section{Histological Studies}

To assess brain damage, $4 \mu \mathrm{m}$-wide Nissl-stained coronal sections were studied as reported elsewhere $(4,12,13)$.

To assess lung damage, $4 \mu \mathrm{m}$-wide sections were stained with hematoxylin-eosin, as described elsewhere (4). A severity-based scoring system ranging from 0 to 3 that assesses atelectasis, edema, and inflammation among other variables (4) was applied by a skilled researcher blinded to the experimental groups.

\section{BALF Collection and Processing}

BALF was obtained at baseline and after being killed, following a standardized procedure described elsewhere (4). Total protein content in BALF was determined in the supernatant using a Pierce BCA Protein Assay Kit (Thermo Scientific, Waltham, MA) according to the manufacturer's instructions.

\section{Assessment of Cytokine Expression in Brain and Lung Tissue}

Samples of frozen brain or lung ( $30 \mathrm{mg}$ ) were obtained to determine IL-1 concentration, as reported elsewhere $(4,12)$. Tissue proteins were quantified by a Pierce BCA Protein Assay Kit (Thermo Scientific), and protein concentration was adjusted to $500 \mu \mathrm{g} / \mathrm{ml}$ per well. IL-1 was then measured by a microarray panel specific for swine tissue (Quantibody Porcine Cytokine Array, RayBiotech, Atlanta, GA). Microarrays were scanned at the Madrid Science Park in Spain.

\section{Assessment of Oxidative Stress}

The presence of protein carbonyl groups was quantified in $15 \mu \mathrm{g}$ of total protein samples from frozen brain and lung tissue using the OxyBlot protein oxidation detection kit (Millipore Iberica, Madrid, Spain), as described elsewhere $(12,13)$. Membranes were incubated with primary antibody $(1: 150)$ dilution for $1 \mathrm{~h}$ and then with secondary antibody (1:300) for $1 \mathrm{~h}$ at room temperature. Finally, the peroxidase reaction was developed with an Enhanced Chemiluminescence Kit (GE Healthcare, Buckinghamshire, UK). Films were scanned and analyzed with ImageJ $1.43 \mathrm{u}$ software (US National Institute of Health, Bethesda, MD, USA). Protein oxidation levels were quantified by densitometric analysis and normalized by total protein loading (Red Ponceau staining), and expressed by the OxyBlot/Red Ponceau ratio $(12,13)$.

\section{Statistical Analysis}

StatPlus:mac2009 software (AnalystSoft, Walnut, CA, USA) was used for all statistical analyses. All data are presented as mean \pm s.e. Mean values were compared using the Mann-Whitney test. For multiple comparison, mean values were compared using the Kruskall-Wallis test for range analysis with Dunn's post hoc test for multiple comparisons. $P<0.05$ was considered to be statistically significant.

\section{ACKNOWLEDGMENTS}

Technical and human support provided by María Dolores Molina Corzo is gratefully acknowledged. We are also indebted to Francisco Tendillo, MD, $\mathrm{PhD}$, for his help with performing this experiment. Finally, we thank Jason Willis-Lee MITI for author's editing services provided during preparation of the final manuscript.

\section{STATEMENT OF FINANCIAL SUPPORT}

This work was supported by grants from the Research Institute Carlos III (ISCIII) according to the Spanish Plan for RI+D+I 2008-2011, the Spanish Plan for Scientific and Technical Research and Innovation 2013-2016, with co-funding from European Regional Development Funds (FEDER) (FIS PI12/00852, PS12/00192, and PI13/01722), the Biomedicine Program, Community of Madrid (S2010/BMD-2308), and GW Research (Cambridge, UK; GWCRI09119). None of the funding sources were involved in the study design, collection, analysis, and interpretation of data or the decision to submit the article for publication.

Disclosure: Jose Martinez-Orgado has a Research Agreement with GW Research (Cambridge, UK). The remaining authors declare no conflict of interest.

\section{REFERENCES}

1. Martín-Ancel A, García-Alix A, Gayá F, Cabañas F, Burgueros M, Quero J. Multiple organ involvement in perinatal asphyxia. J Pediatr 1995;127:786-93.

2. Shah P, Riphagen S, Beyene J, Perlman M. Multiorgan dysfunction in infants with post-asphyxial hypoxic-ischaemic encephalopathy. Arch Dis Child Fetal Neonatal Ed 2004;89:F152-5.

3. Bocking AD, Gagnon R, White SE, Homan J, Milne KM, Richardson BS. Circulatory responses to prolonged hypoxemia in fetal sheep. Am J Obstet Gynecol 1988;159:1418-24.

4. Arruza L, Pazos MR, Mohammed N, et al. Hypoxic-ischemic brain damage induces distant inflammatory lung injury in newborn piglets. Pediatr Res 2015;79:401-8.

5. Hampson AJ, Grimaldi M, Axelrod J, Wink D. Cannabidiol and (-) Delta9-tetrahydrocannabinol are neuroprotective antioxidants. Proc Natl Acad Sci USA 1998;95:8268-73.

6. Mechoulam R, Peters M, Murillo-Rodriguez E, Hanus LO. Cannabidiolrecent advances. Chem Biodivers 2007;4:1678-92.

7. Pertwee R. The pharmacology and therapeutic potential of cannabidiol. In: Di Marzo V, ed. Cannabinoids. New York: Kluwer Academic/Plenum Publishers, 2004:32-83.

8. Castillo A, Tolón MR, Fernández-Ruiz J, Romero J, Martinez-Orgado J. The neuroprotective effect of cannabidiol in an in vitro model of newborn hypoxic-ischemic brain damage in mice is mediated by CB2 and adenosine receptors. Neurobiol Dis 2010;37:434-40.

9. Alvarez FJ, Lafuente H, Rey-Santano MC, et al. Neuroprotective effects of the nonpsychoactive cannabinoid cannabidiol in hypoxic-ischemic newborn piglets. Pediatr Res 2008;64:653-8.

10. Lafuente H, Alvarez FJ, Pazos MR, et al. Cannabidiol reduces brain damage and improves functional recovery after acute hypoxia-ischemia in newborn pigs. Pediatr Res 2011;70:272-7.

11. Pazos MR, Cinquina V, Gómez A, et al. Cannabidiol administration after hypoxia-ischemia to newborn rats reduces long-term brain injury and restores neurobehavioral function. Neuropharmacology 2012;63:776-83.

12. Pazos MR, Mohammed N, Lafuente H, et al. Mechanisms of cannabidiol neuroprotection in hypoxic-ischemic newborn pigs: role of $5 \mathrm{HT}(1 \mathrm{~A})$ and CB2 receptors. Neuropharmacology 2013;71:282-91. 


\section{Articles | Arruza et al.}

13. Lafuente H, Pazos MR, Alvarez A, et al. Effects of cannabidiol and hypothermia on short-term brain damage in new-born piglets after acute hypoxia-ischemia. Front Neurosci 2016;10:323.

14. Russo EB, Burnett A, Hall B, Parker KK. Agonistic properties of cannabidiol at 5-HT1a receptors. Neurochem Res 2005;30:1037-43.

15. Mishima K, Hayakawa K, Abe K, et al. Cannabidiol prevents cerebral infarction via a serotonergic 5-hydroxytryptamine1A receptor-dependent mechanism. Stroke 2005;36:1071-6.

16. Ribeiro A, Ferraz-de-Paula V, Pinheiro ML, et al. Cannabidiol, a nonpsychotropic plant-derived cannabinoid, decreases inflammation in a murine model of acute lung injury: role for the adenosine $\mathrm{A}(2 \mathrm{~A})$ receptor. Eur J Pharmacol 2012;678:78-85.

17. Ribeiro A, Almeida VI, Costola-de-Souza C, et al. Cannabidiol improves lung function and inflammation in mice submitted to LPSinduced acute lung injury. Immunopharmacol Immunotoxicol 2015;37: $35-41$.

18. Muhammad S, Barakat W, Stoyanov S, et al. The HMGB1 receptor RAGE mediates ischemic brain damage. J Neurosci 2008;28:12023-31.

19. Liesz A, Dalpke A, Mracsko E, et al. DAMP signaling is a key pathway inducing immune modulation after brain injury. J Neurosci 2015;35: 583-98.

20. Koutsoukou A, Katsiari M, Orfanos SE, et al. Respiratory mechanics in brain injury: a review. World J Crit Care Med 2016;5:65.

21. Hayakawa K, Irie K, Sano K, et al. Therapeutic time window of cannabidiol treatment on delayed ischemic damage via high-mobility group box1-inhibiting mechanism. Biol Pharm Bull 2009;32:1538-44.
22. Vuolo F, Petronilho F, Sonai B, et al. Evaluation of serum cytokines levels and the role of cannabidiol treatment in animal model of asthma. Mediators Inflamm 2015;2015:538670.

23. Kim YE, Park WS, Sung DK, et al. Intratracheal transplantation of mesenchymal stem cells simultaneously attenuates both lung and brain injuries in hyperoxic newborn rats. Pediatr Res 2016;80:415-24.

24. Seeley EJ. A dry lung is a happy lung: More supporting evidence. J Thorac Cardiovasc Surg 2015;149:321-2.

25. Johnston MV, Fatemi A, Wilson MA, Northington F. Treatment advances in neonatal neuroprotection and neurointensive care. Lancet Neurol 2011;10:372-82.

26. Gdovin MJ, Zamora DA, Ravindran CRM, Leiter JC. Serotonergic modulation of respiratory rhythmogenesis and central chemoreception. Ethn Dis 2010;20:S1-39-44.

27. Barrett KT, Kinney HC, Li A, Daubenspeck JA, Leiter JC, Nattie EE. Subtle alterations in breathing and heart rate control in the 5-HT1A receptor knockout mouse in early postnatal development. J Appl Physiol 2012;113:1585-93.

28. Skinner MR, Ramage AG, Jordan D. Modulation of reflexly evoked vagal bradycardias by central 5-HT1A receptors in anaesthetized rabbits. Br J Pharmacol 2002;137:861-73.

29. Ramage AG, Villalón CM. 5-Hydroxytryptamine and cardiovascular regulation. Trends Pharmacol Sci 2008;29:472-81.

30. Dudášová A, Keir SD, Parsons ME, Molleman A, Page CP. The effects of cannabidiol on the antigen-induced contraction of airways smooth muscle in the guinea-pig. Pulm Pharmacol Ther 2013;26:373-9. 